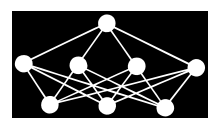

\title{
IDENTIFICATION OF TYPE DAILY \\ DIAGRAMS OF ELECTRIC CONSUMPTION \\ BASED ON CLUSTER ANALYSIS \\ OF MULTI-DIMENSIONAL DATA BY \\ NEURAL NETWORK
}

Bohumír Garlík, Miloš Křivan ${ }^{\dagger}$

\begin{abstract}
This article establishes a mathematical description of a self-organizing neural network used for cluster analysis with a subsequent sampling of its effectiveness as an example of identification of the type daily diagrams of electric energyconsumption of complex intelligent buildings within an electric micro grid, namely for a typical work day and a day off on the basis of its annual history. The mentioned type daily diagram can be used to predict power consumption. This method is given in the context of the commonly used procedure for cluster analysis. The experiment was processed in the computer program Artint (C) 2010.
\end{abstract}

Key words: Artificial Neural Network (ANN), Self-Organizing Map (SOM), Kohonen map, counter-propagation neural network, cluster analysis, Type Daily Diagrams (TDD), load prediction, intelligent building, micro grid

Received: March 3, 2013

Revised and accepted: June 4, 2013

\section{Introduction}

With the development of computing technology and the growth of its computational power, there has been an increasing focus on artificial intelligence methods since World War 2. These methods include terms such as artificial neural networks, fuzzy

\footnotetext{
*Bohumír Garlík

Department of Microenvironmental and Building Services Engineering, Czech Technical University in Prague, Thákurova 7, 16000 Prague 6, E-mail: bohumir.garlik@fsv.cvut.cz; Department of Management and Artificial Intelligence, Research Institute for Intelligent Buildings, Brno, Drobného 16, 60200 Brno, Czech Republic, , E-mail: bohumir.garlik@vuib.cz

† Miloš Křivan

Department of Management and Artificial Intelligence, Research Institute for Intelligence Buildings, Brno, Drobného 16, 60200 Brno, Czech Republic, E-mail: pwrnetexp@gmail.com
} 
sets or evolutionary algorithms. However, their massive utilization in practical applications across all human activities only occurred in the eighties of the previous century, due to the development of personal computers.

Artificial neural networks are used to process and evaluate incomplete, indeterminate or inconsistent information, especially for tasks involving recognition, diagnostics, classification of objects with respect to provided categories or prediction of the time development of the given variable, compression and coding information, noise filtering, extrapolation or interpolation of the trends of a given variable and last but not least the cluster analysis of multidimensional data, as described in this article. More precisely, by recognition we mean the recognition of visual or acoustic information, such as written text or spoken words, and by diagnostics we mean diagnostics of the residual service life of technological equipment or human organisms including bio-medicinal signals such as ECG or EEG.

\section{Competitive Model of Neural Network}

We define an artificial neural network as the oriented graph with edges and vertices rated dynamically, i.e. as the ordered quintuplet $[V, E, \varepsilon, w, y]$ :

$V \quad$ set of vertices (neurons)

$E \quad$ set of edges (synapses)

$\varepsilon \quad$ mapping edges with incidence vertices $(\varepsilon: E \rightarrow V \times V)$

$w \quad$ dynamic valuation of edges $(w: \varepsilon(E) \times \mathbf{T} \rightarrow \mathbb{R})$

$y$ dynamic valuation of vertices $(y: V \times \mathbf{t} \rightarrow \mathbb{R})$.

The vector $\vec{w}(T)=\left[w_{i j}(T) \mid[i, j] \in V \times V\right]$ is called the network configuration in time $T,\left(\forall[i, j] \notin \varepsilon(E) \Rightarrow w_{i j}(T)=0\right)$ and the vector $\vec{y}(t)=\left[y_{i}(t) \mid i \in V\right]$ is called the network state in time $t$. The configuration respectively state of the network as a vector function of time $T$ or $t$ is referred to as adaptive dynamics respectively active dynamics of the neural network. Active or adaptive dynamics of a neural network in continuous time can be defined as a vector solution of the following systems of differential equations [1]:

$$
\frac{d}{d t} x_{j}(t)+x_{j}(t)=\sum_{i} f_{i}\left(x_{i}(t-\Delta t)\right) w_{i j}-\vartheta_{j}
$$

respectively

$$
\frac{d}{d T} w_{i j}(T)+\beta g_{j}\left(x_{j}(T)\right) w_{i j}(T)=\alpha f_{i}\left(x_{i}(T)\right) g_{j}\left(x_{j}(T)\right)
$$

$i, j \in V, \alpha, \beta \in\langle 0,1\rangle$, and then analogously to biological neural network we have:

$x_{i} \quad$ potential of the $i$-th neuron

$f_{i} \quad$ activation function of the $i$-th neuron $\left(f_{i}\left(x_{i}\right)=y_{i}\right)$

$g_{j} \quad$ adaptation function of the $j$-th neuron

$\vartheta_{j} \quad$ threshold of the $j$-th neuron

$w_{i j} \quad$ synaptic weight links of the $i$-th neuron to the $j$-th neuron 
$\alpha \quad$ measure of plasticity of synapses

$\beta \quad$ measure of elasticity of synapses

$\Delta t \quad$ signal delay time. time:

If we replace in (1) and (2) the derivations by analogous expressions for discrete

$$
\frac{d}{d t} x_{j}(t) \equiv \frac{x_{j}(t+1)-x_{j}(t)}{t+1-t} \quad \frac{d}{d T} w_{i j}(T) \equiv \frac{w_{i j}(T+1)-w_{i j}(T)}{T+1-T}
$$

and if we set $\Delta t=0$, then we obtain the following systems of difference equations and the vectors of its solutions define the active and adaptive dynamics of a neural network in discrete time:

$$
x_{j}(t+1)=\sum_{i} f_{i}\left(x_{i}(t)\right) w_{i j}-\vartheta_{j} \quad y_{j}(t+1)=f_{j}\left(\sum_{i} y_{i}(t) w_{i j}-\vartheta_{j}\right)
$$

respectively

$$
w_{i j}(T+1)=\left(1-\beta g_{j}\left(x_{j}(T)\right)\right) w_{i j}(T)+\alpha f_{i}\left(x_{i}(T)\right) g_{j}\left(x_{j}(T)\right)
$$

$i, j \in V$.

We approximate the dependence of the state on the potential of the neuron by sigmoid function: $f(x)=\frac{1}{\left(1+e^{-p x}\right)}$ where the parameter $p>0$ expresses the slope of the sigmoid. For a slope approaching zero or infinity we get the activation function in the shape of linearity respectively non-linearity:

$$
\lim _{p \rightarrow 0} f(x)=\frac{1}{2} \quad \lim _{p \rightarrow \infty} f(x)=0 \quad x<0 \quad \lim _{p \rightarrow \infty} f(x)=1 \quad x>0
$$

and we can finally define the following network function: $\vec{F}(\vec{x}(t))=\vec{y}(t+\Delta t)$, where $\Delta t$ is the response time of the network.

Let us divide the population of the neurons in $V$ to two disjoint populations $V_{1}$ and $V_{2}\left(V_{1} \cup V_{2}=V, V_{1} \cap V_{2}=\emptyset,\left|V_{1}\right|=n,\left|V_{2}\right|=m\right)$, and let us connect them by edges so that there is an edge from each neuron in $V_{1}$ to each neuron in $V_{2}$ $\left(\varepsilon\left(E_{1}\right)=V_{1} \times V_{2}\right)$, i.e. the network is oriented from $V_{1}$ to $V_{2}$ and $V_{1}$ respectively $V_{2}$ is then understood as the input respectively output population. Let us, furthermore, connect neurons in $V_{2}$ by edges so that there is an edge from each neuron in $V_{2}$ to every other neuron in $V_{2}\left(\varepsilon\left(E_{2}\right)=V_{2} \times V_{2}-\left\{[j, j] \mid j \in V_{2}\right\}\right)$.

Let us choose the activation function of neurons of population $V_{1}$ as an identity, i.e. modified linearity and the activation function of neurons of population $V_{2}$ as non-linearity. Then, during the active dynamics for constantly applied stimulus attached to population $V_{1}$ we can express the active dynamics (3) for $y_{k}(0)=0$ as follows:

$$
y_{j}(t+1)=f_{j}\left(\sum_{k} y_{k}(t) w_{k j}-\vartheta_{j}\right) \quad-\vartheta_{j}=\sum_{i} x_{i}(0) w_{i j}
$$


$i \in V_{1}, j, k \in V_{2}$ and let us call the parameter $-\vartheta_{j}$ the gain potential of the $j$-th neuron.

Let us choose the following initial conditions for the network configuration $w_{k j}(0)=-2, w_{i j}(0)=r_{i j}$, and let us add templates from the training set specified in the form $\{\vec{a}(T) \mid T \in \Delta T\}$ for the population $V_{1}$, where $\Delta T$ is the network adaptation period. If we only let the mutual links between neurons in $V_{1}$ and $V_{2}$ adapt, and if we select the adaptation function for the neurons in $V_{2}$ to match the activation functions, then, assuming elasticity is equal to plasticity $(\alpha=\beta)$, we can express the adaptive dynamics (4) as follows:

$$
\begin{gathered}
w_{k j}(T)=w_{k j}(T-1) \\
w_{i j}(T)=w_{i j}(T-1)+\alpha y_{j}(T)\left(a_{i}(T)-w_{i j}(T-1)\right)
\end{gathered}
$$

$i \in V_{1}, j, k \in V_{2}, T \in \Delta T=\{1,-, N\}$, where $N$ respectively $r_{i j}$ is the number of patterns of training set respectively the value specified of the random number generator.

In each step of the adaptive dynamics (6) it is required to designate the states of neurons in $V_{2}$, i.e. the steps of the adaptive dynamics are conditioned by the active dynamics, which, from the perspective of adaptive dynamics, runs infinitely fast. Thus, the state of $V_{2}$ is determined synchronously with the state of $V_{1}$.

Let us assign to each neuron in $V_{2}$ a weight vector $\vec{w}_{j}=\left[w_{i j} \mid i \in V_{1}\right]$. Then the neurons in $V_{2}$ together with the edges $E_{2}$ and the active dynamics (5) form a Hopfield optimization network [4] with the following energy function:

$$
E(\vec{y})=\sum_{j} \sum_{k} y_{k} y_{j}+\sum_{j} y_{j} \vartheta_{j} \quad-\vartheta_{j}=\sum_{i} a_{i}(T) w_{i j}=\vec{a}(T) \cdot \vec{w}_{j}
$$

$i \in V_{1}, j \in V_{2}, k \in V_{2}-\{j\}$.

If the vectors of the training set respectively the weight vectors are normal, then the received potential of each neuron will comply with $-\vartheta=\cos \varphi$ and the distance between the specified vectors can be defined as the angle $\varphi \in\langle 0, \pi\rangle$ between them. The energy function specified above will then reach its minimum if and only if only one neuron in $V_{2}$ is excited, specifically the neuron with the maximum potential gain (7) - the so-called gain neuron.

The process of energy minimization of the state of $V_{2}$ realized by the active dynamics (5), when the excited neuron with the maximum potential gain inhibits (by negative links) other neurons, is called lateral inhibition. Lateral inhibition, which designates a corresponding state of the population of $V_{2}$ based on the presented training template, replaces the missing template association in the training set - in other words, it replaces the statement of a teacher, and we thus speak of teacher-less learning.

Lateral inhibition in each adaptation step will ensure the adaptation of only the weight vector corresponding to the $k$-th gain neuron, i.e. of the weight vector as per the above-specified non-Euclidean metric of the closest presented training set template, to which it will advance on the surface of an $n$-dimensional ball of unit radius by an adaptation step proportional to the plasticity of the synapse (Fig. 1): 


$$
\vec{w}_{k}(T)=\vec{w}_{k}(T-1)+\alpha\left(\vec{a}(T)-\vec{w}_{k}(T-1)\right)
$$

and the gain neuron thus won the competition for the presented template of the training set. The normality of the adapted weight vector will be ensured by its subsequent normalization.

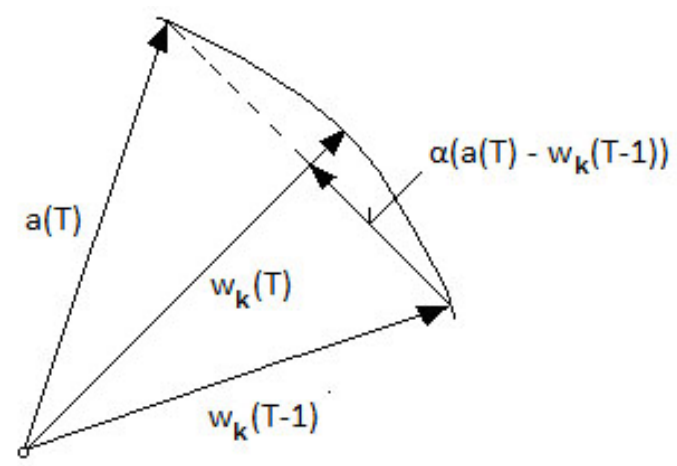

Fig. 1 Adaptation step.

The objective function (9) will reach its minimum if and only if the weight vector is on the position with a minimal sum of distances from all vectors of the training set which excite the appropriate neuron, i.e. in the center of the cluster of the specified vectors:

$$
G\left(\vec{w}_{j}\right)=\frac{1}{2} \sum_{T} y_{j}(T) \sum_{i}\left(a_{i}(T)-w_{i j}\right)^{2} \quad-\frac{\partial G\left(\vec{w}_{j}\right)}{\partial w_{i j}}=\sum_{T} y_{j}(T)\left(a_{i}(T)-w_{i j}\right)
$$

$i \in V_{1}, j \in V_{2}, T \in \Delta T$.

Adaptive dynamics (6) is a gradient descent on a lower-bounded objective function (9), and so, assuming that the vectors of the training set form clusters in the $n$-dimensional space whose size corresponds to the cardinality of $V_{2}$, the (initially randomly located) weight vectors will converge towards the centers of these clusters during adaptive dynamics.

Let us define the following categories of normal vectors:

$$
C_{k}=\left\{\vec{x} \in \Omega \mid \varphi\left(\vec{x}, \vec{w}_{k}\right)<\varphi\left(\vec{x}, \vec{w}_{j}\right)\right\} \quad \Omega=\left\{\vec{x} \in \mathbb{R}^{n} \| \vec{x} \mid=1\right\}
$$

$k \in V_{2}, j \in V_{2}-\{k\}$ and $\varphi$ is a non-Euclidean metric, i.e. the angle between the vectors.

The function of the network will thus assign, during lateral inhibition, a vector of the canonical basis of an $m$-dimensional space with a one on the $k$-th position to an arbitrary normal network input, if and only if the network input lies in the $k$-th category (10). The function of the network of the competitive model can thus be understood as a classification with respect to the categories specified above. 
If we set $\left|V_{2}\right|=m^{2}$, then we can interpret the neurons in $V_{2}$ as elements of a square $m \times m$ grid. Let us define the square neighborhood of the $r$-th order of the $k$-th element of the grid as the set containing all grid elements which lie at a distance of less than or equal to order $r$, i.e. $\sigma(k, r)=\left\{j \in V_{2} \mid \rho(k, j) \leq r\right\}$, where $\rho$ is the metric defined on the grid as the neighborhood of elements of the appropriate order, and let us adjust the adaptive dynamics (8) for the $k$-th gain neuron:

$$
\vec{w}_{j}(T)=\vec{w}_{j}(T-1)+\alpha_{j}(T)\left(\vec{a}(T)-\vec{w}_{j}(T-1)\right)
$$

$j \in \sigma(k, r)$ and the plasticity drops globally with the time of the adaptive dynamics and locally with the order of the distance of the appropriate neuron from the gain neuron in the population grid of $V_{2}$.

The adjustment of the adaptive dynamics specified above generalize lateral inhibition by the extension of the excitation of the gain neuron to its neighborhood, which links the above-specified metric $\varphi$ with the above-specified metric $\rho$. If the vectors of the training set are randomly distributed in the $n$-dimensional space in accordance with some distribution function, then after the adaptation of the network the weight vectors will be randomly distributed in the same area in accordance with the same distribution function.

If we present a training set on an adapted network in active mode, then the map of the frequency of excitations of neurons in $V_{2}$, the so-called Kohonen map [2] will provide a mapping of the clusters of vectors of the training set in an $n$ dimensional space. Such a generalized competitive model, under assumption of a sufficiently large cardinality of $V_{2}$, performs the cluster analysis of the training set, i.e. determines the number of clusters and their distribution in the $n$-dimensional space.

Let us adjust the topology of the already adapted competitive model by adding a population set $V_{3}$, connected by edges to the population $V_{2}$ so that there is an edge from each neuron in $V_{2}$ to each neuron in $V_{3}\left(\varepsilon\left(E_{3}\right)=V_{2} \times V_{3}\right)$. Let the new output population $V_{3}$ have the same cardinality as the input population $V_{1}$, and thus the population $V_{2}$ becomes a hidden population.

Let us set the weights of edges $E_{3}$ as follows: $w_{j q(i)}=w_{i j}, i \in V_{1}, j \in V_{2}, q(i) \in$ $V_{3}$, where $q(i)$ is the image of the $i$-th neuron of population $V_{1}$ in population $V_{3}$. The output population $V_{3}$ together with the weighted edges $E_{3}$ thus forms an image of the output population $V_{1}$ together with the weighted edges $E_{1}$ mirrored over the hidden population $V_{2}$, a phenomenon which we call counter propagation [3] of the synaptic weights of edges $E_{1}$ to edges $E_{3}$ in the direction of the orientation of edges. Let us select the activation functions of neurons in $V_{3}$ identically to the activation functions of neurons in $V_{1}$. Then, during active dynamics after the stabilization of the state of the population of $V_{2}$, the excitation of the $k$-th gain neuron will lead to the following values of potentials of neurons in $V_{3}$ :

$$
x_{q(i)}=\sum_{j} y_{j} w_{j q(i)}=w_{k q(i)}=w_{i k}
$$


$i \in V_{1}, j \in V_{2}$, then stimulus $\vec{x} \in C_{k}$ implies the following network function: $\vec{F}(\vec{x})=\vec{w}_{k}$.

The function of the network in the competitive model with forward propagation of weights will thus assign a prototype (the closest weight vector) to each normal network input. Prototypes lie in the centers of the appropriate clusters and thus represent these clusters - they are their typical representatives.

The competitive model with forward propagation of weights and the Kohonen map may be used to reduce the cardinality of multidimensional data, which may be replaced by a set of prototypes of their elements with cardinality of $m^{2}$.

\section{Experiment}

The goal of this experiment is the identification of type daily diagrams of hourly consumption of electric energy by a complex of intelligent buildings within an electric micro-grid on a workday in the middle of the workweek, i.e. Wednesday, and on a non-work day, specifically using a non-work day before a non-work day and also a non-work day before a work day, i.e. on Saturday and Sunday, based on the recorded annual history of hour consumption of electric energy by the complex.

To allow the measuring of the efficiency of the utilized cluster analysis method, the annual history of hourly consumption of electric energy has been artificially modeled so that a typical daily diagram may be compared to a certain standard. The default standards of daily diagrams of hourly consumptions were the characteristic hourly developments of the consumption of the above-listed three days, where each hourly consumption of each of these was randomly modified by a random number generator with a normal probability distribution, as many times as was necessary to fill the annual history of hourly consumption, i.e. two hundred sixty times for Wednesday and fifty two times for Saturday and Sunday each, i.e. three hundred sixty four daily diagrams in total. A demonstration of the source code, in Fortran, of the used computer program is shown below:

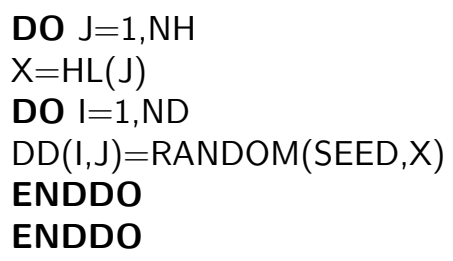

!NH - NUMBER OF HOURS (24)

!HL - HOURLY LOAD

!ND - NUMBER OF DAYS (260/52)

!DD - YEAR HISTORY OF DAILY DIAGRAMS 


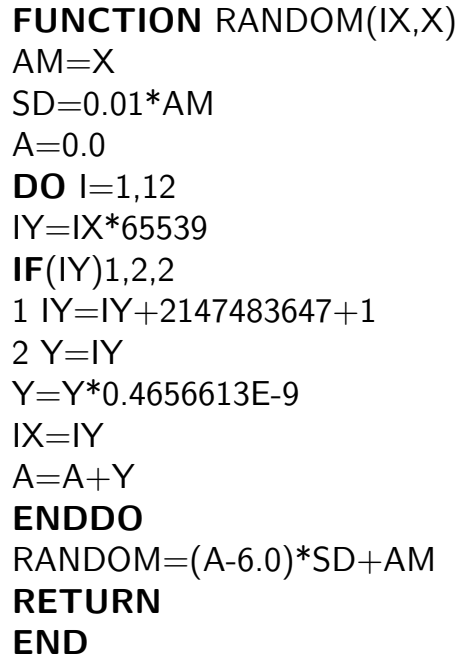

The attached images each contain four examples of randomly modeled daily diagrams of Saturday (Fig. 2), Sunday (Fig. 3) and Wednesday (Fig. 4).

This model of the annual history of hourly consumption of energy, i.e. a rectangular matrix with 364 rows and 24 columns, which represents our multidimensional data, then forms the training set during adaptive dynamics presented to the competitive model of the artificial neural network on a day-by-day basis, i.e. the input always being one day containing 24 attributes. The topology of the network used is then formed by two layers of neurons: the input layer with 24 neurons and the output layer with 225 neurons, which is organized in a square 15 by 15 grid. The plasticity dropped exponentially during network adaptation, from the default value of 1 to its final value of 0.005 , and the order of the neighborhood of the gain neuron during network adaptation dropped exponentially from its default value of 7 to the final value of 0 , i.e. the neighborhood of the gain neuron of the default order covered the whole output layer of the network and by the end of the network adaptation it degenerated to only contain the gain neuron.

After network adaptation, the active dynamics process was initiated by representing the training samples. This has led to the appearance of a Kohonen Map (Fig. 5), which depicts three well-separated clusters, two of which are smaller and likely correspond to Saturday and Sunday, and one of which is larger and likely corresponds to Wednesday. The map may be interpreted as a map of the landscape with a marked elevation (above sea-level) for each spot, where each island represents a cluster of daily diagrams and the highest point, marked by a white circle on the figure, represents the prototype representing the given cluster, i.e. the type daily diagram of the appropriate period. The map should then be understood as the surface of a globe, i.e. the top respectively left rim is identified with the bottom respectively top rim. Neurons with a zero number of excitations correspond to weight vectors which were never the closest to any of the presented templates in the training set, i.e. during adaptive dynamics these did not come sufficiently close to the clusters of training templates. Forward propagation may be used to extract the necessary weight vectors from the configuration of the learned network during active dynamics. These results in the sought daily diagrams are provided 
Garlík B., Křivan M.: Identification of type daily diagrams...
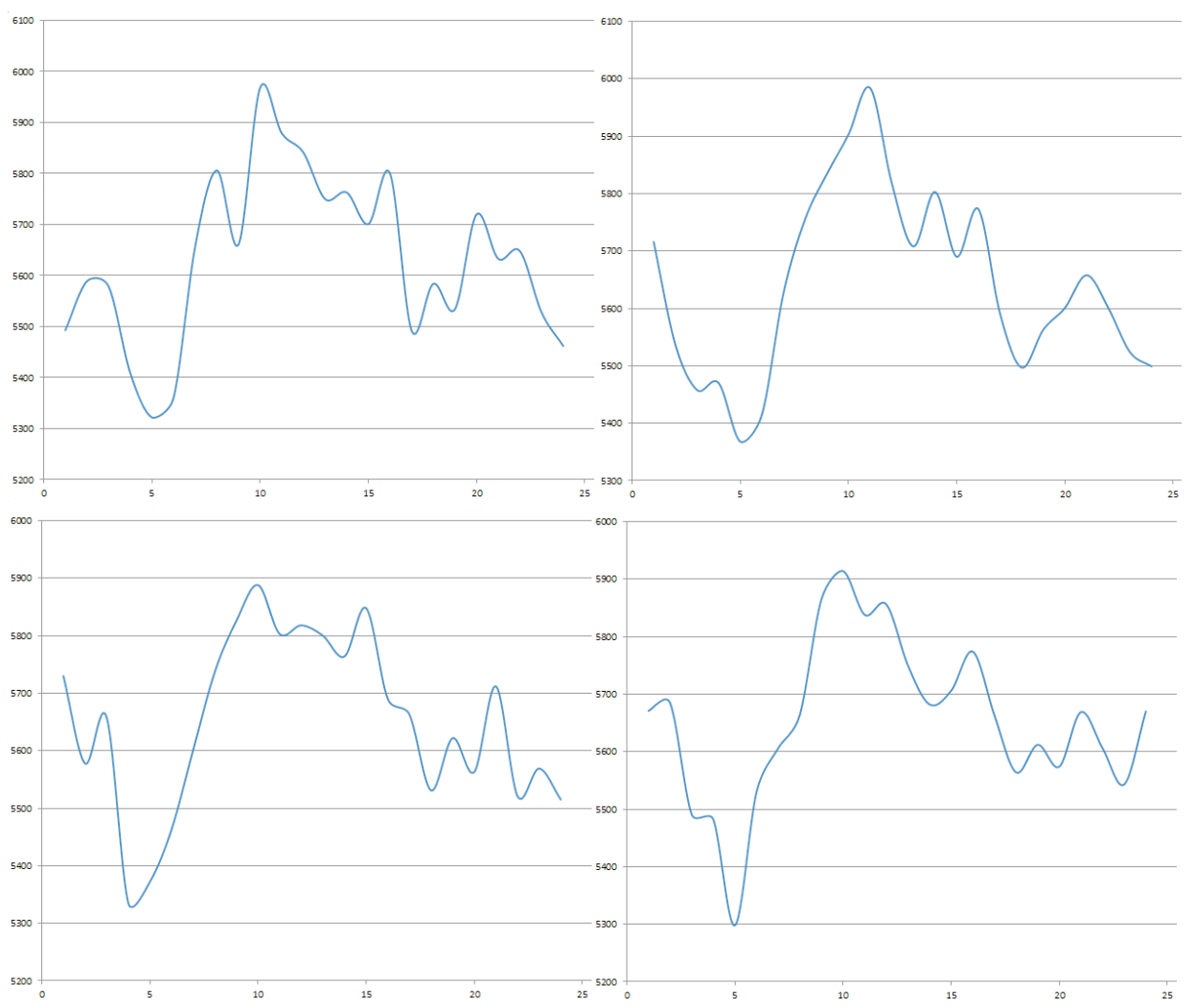

Fig. 2 Examples of randomly modeled diagrams of Saturday.

in Figs. 6-8 together with a comparison to the appropriate standard.

Although individual daily diagrams of the annual history (Figs. 2-4) are mutually relatively different, and for instance the fourth example of a Saturday daily diagram (Fig. 2) is more similar to a characteristic Sunday daily diagram, the resulting type daily diagrams are very similar to the appropriate standards (Figs. 6-8). This documents the high efficiency of the utilized cluster analysis method. Tab. I contains a numerical comparison of the type daily diagrams with the appropriate standards, and their average respectively maximum deviation is $0.2 \%$ respectively $0.5 \%$.

\section{Conclusion}

The mathematical description provided above and the experiment which was carried out implies that the cluster analysis method used in the article clearly fulfills the five generally formulated attributes of an ideal method:

- The method does not require any a priori information from the user 


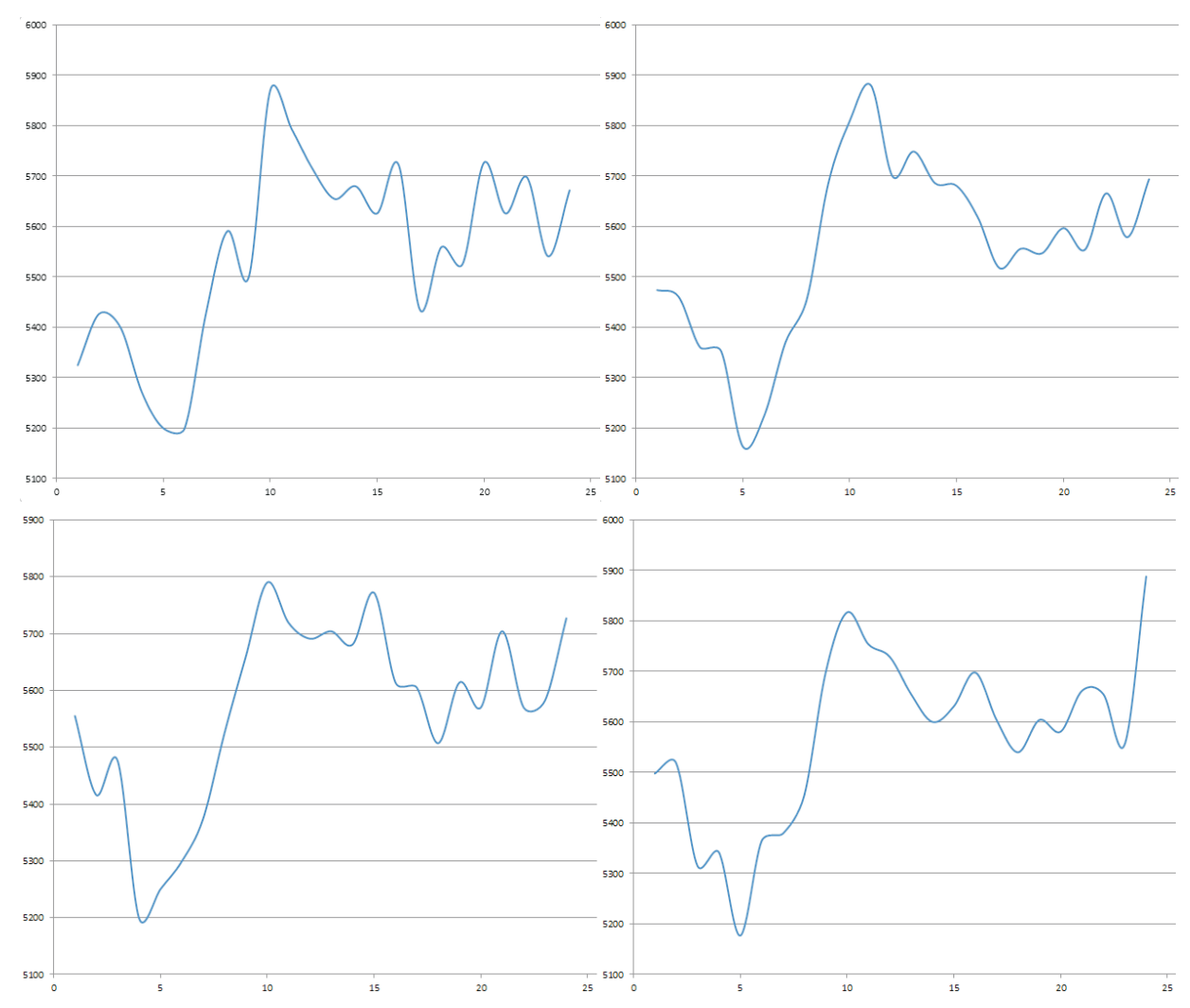

Fig. 3 Examples of randomly modeled diagrams of Sunday.

- The method identifies clusters of arbitrary shape and density of contained objects

- The method is not sensitive to the order of the presented observations of objects

- The method is robust towards remote observations of objects

- The method is capable of analyzing a set of a large number of observations with a large number of variables during a single presentation

and, as may be seen from the Kohonen map, it is also hierarchical, since each cluster in the map also contains sub-clusters.

The most frequently used methods of cluster analysis include the non-hierarchical $k$-means method, which is included in basically all statistical programs. Its algorithm is based on an a priori selection of the number of clusters, including random generation of their centroids and subsequent inclusion of individual objects into clusters based on their distance (Euclidean metric) from the centroids, which are continuously updated based on the average values of coordinates of all objects in the given cluster. This method does not comply with even one of the properties 
Garlík B., Křivan M.: Identification of type daily diagrams...

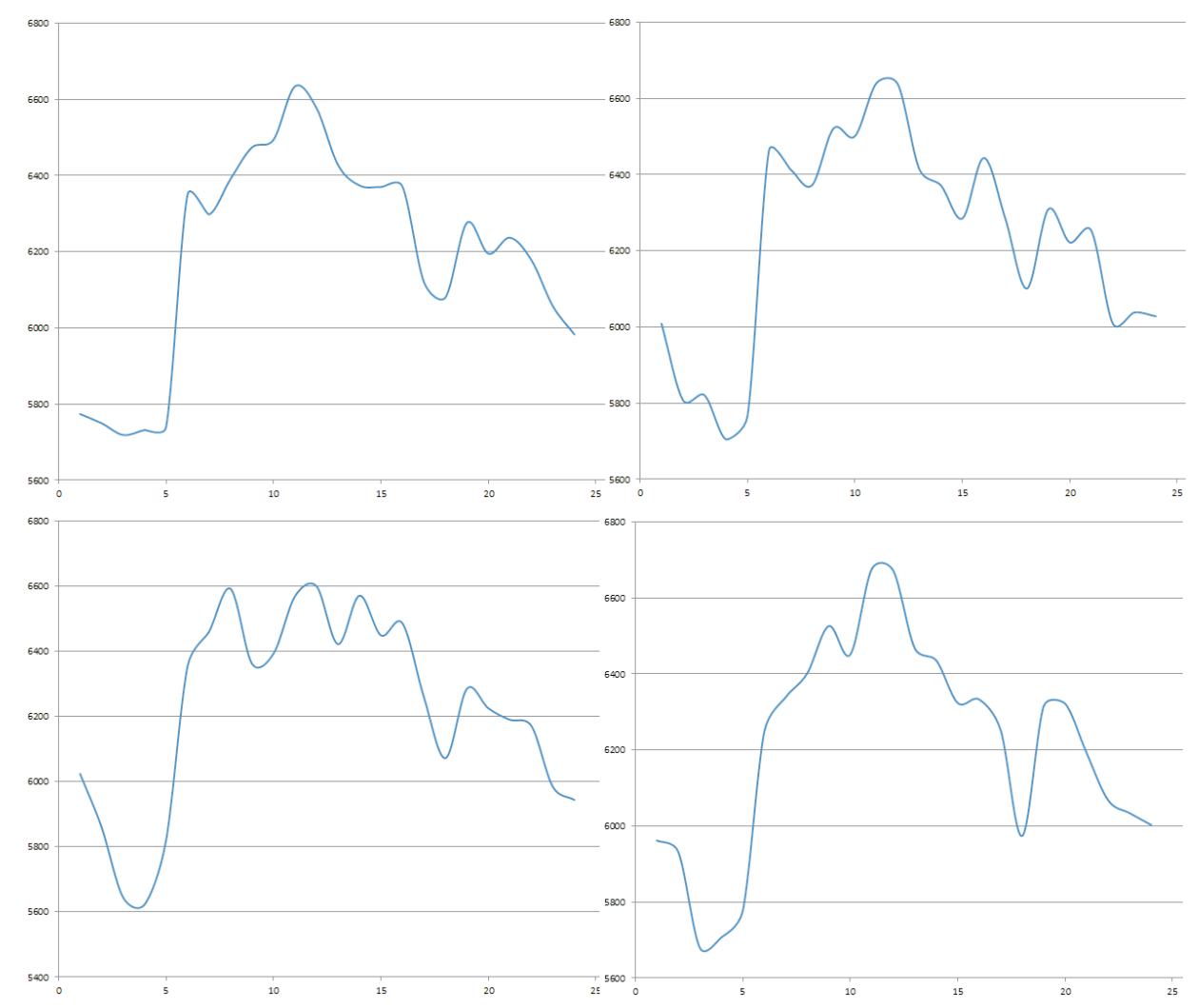

Fig. 4 Examples of randomly modeled diagrams of Wednesday.

\begin{tabular}{|c|c|c|c|c|c|c|c|c|c|c|c|c|c|c|}
\hline 0 & 0 & 0 & 0 & 0 & 0 & 1 & 3 & 1 & 3 & 6 & 2 & 0 & 0 & 0 \\
\hline 0 & 0 & 0 & 0 & 0 & 0 & 4 & 3 & 5 & 2 & 1 & 3 & 0 & 0 & 0 \\
\hline 4 & 3 & 3 & 0 & 0 & 0 & 2 & 2 & 2 & 3 & 0 & 1 & 0 & 0 & 0 \\
\hline 1 & 5 & 2 & 0 & 0 & 0 & 1 & 1 & 6 & 2 & 5 & 2 & 0 & 0 & 0 \\
\hline 2 & 5 & 1 & 0 & 0 & 0 & 7 & 3 & 3 & 1 & 2 & 2 & 0 & 0 & 0 \\
\hline 1 & 7 & 0 & 0 & 0 & 0 & 5 & 1 & 4 & 2 & 7 & 2 & 0 & 0 & 0 \\
\hline 0 & 12 & 0 & 0 & 0 & 2 & 5 & 1 & 8 & 0 & 3 & 3 & 0 & 0 & 0 \\
\hline 0 & $\overline{6}$ & 0 & 0 & 0 & 0 & 0 & 3 & 6 & 0 & 1 & 4 & 0 & 0 & 0 \\
\hline 0 & 0 & 0 & 0 & 0 & 0 & 3 & 0 & 2 & 5 & 0 & 2 & 0 & 0 & 0 \\
\hline 0 & 2 & 0 & 0 & 0 & 1 & 1 & 3 & 0 & 0 & 6 & 2 & 0 & 0 & 0 \\
\hline 0 & 7 & 0 & 0 & 0 & 0 & 8 & 3 & 1 & 2 & 1 & 7 & 0 & 0 & 0 \\
\hline 1 & 6 & 2 & 0 & 0 & 1 & 1 & 2 & 2 & 2 & 4 & 2 & 3 & 0 & 0 \\
\hline 0 & $1 n$ & 1 & 0 & 0 & 0 & 10 & 7 & 2 & 5 & 3 & 7 & 0 & 0 & 0 \\
\hline 1 & 13 & 4 & 0 & 0 & 1 & 5 & 2 & 0 & 0 & 2 & 2 & 0 & 0 & 0 \\
\hline 0 & 4 & 1 & 0 & 0 & 0 & 0 & 7 & 0 & 3 & 1 & 3 & 0 & 0 & 0 \\
\hline
\end{tabular}

Fig. 5 Kohonen map. 


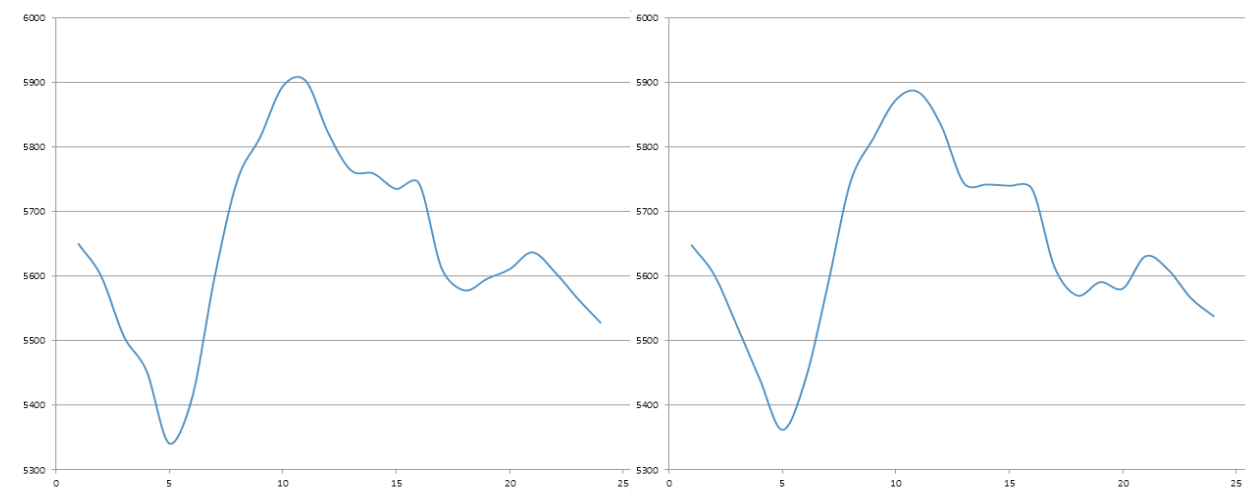

Fig. 6 Type daily diagram and standard of Saturday.

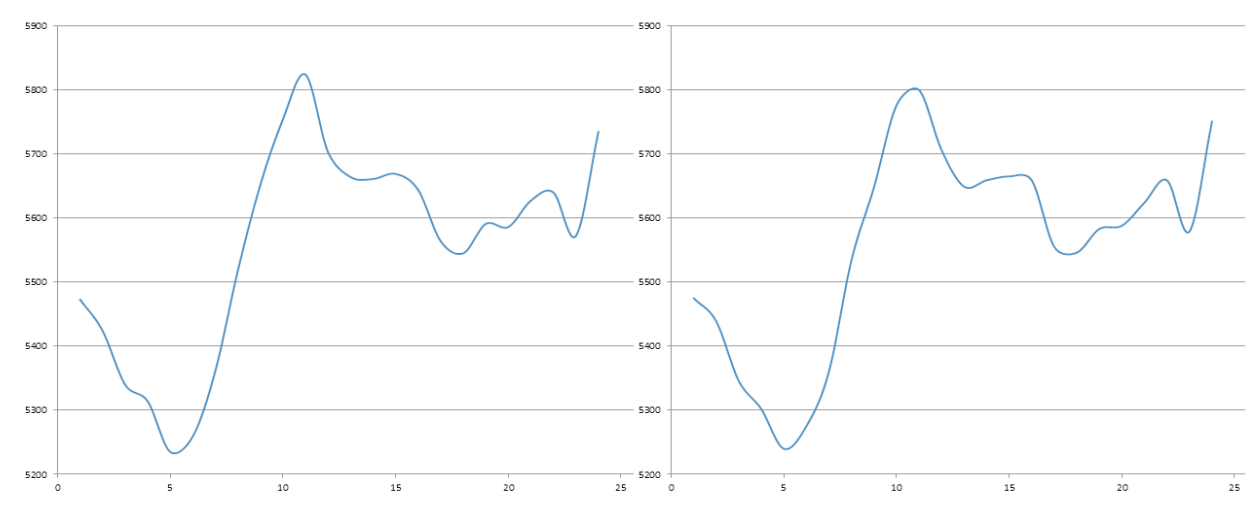

Fig. 7 Type daily diagram and standard of Sunday.

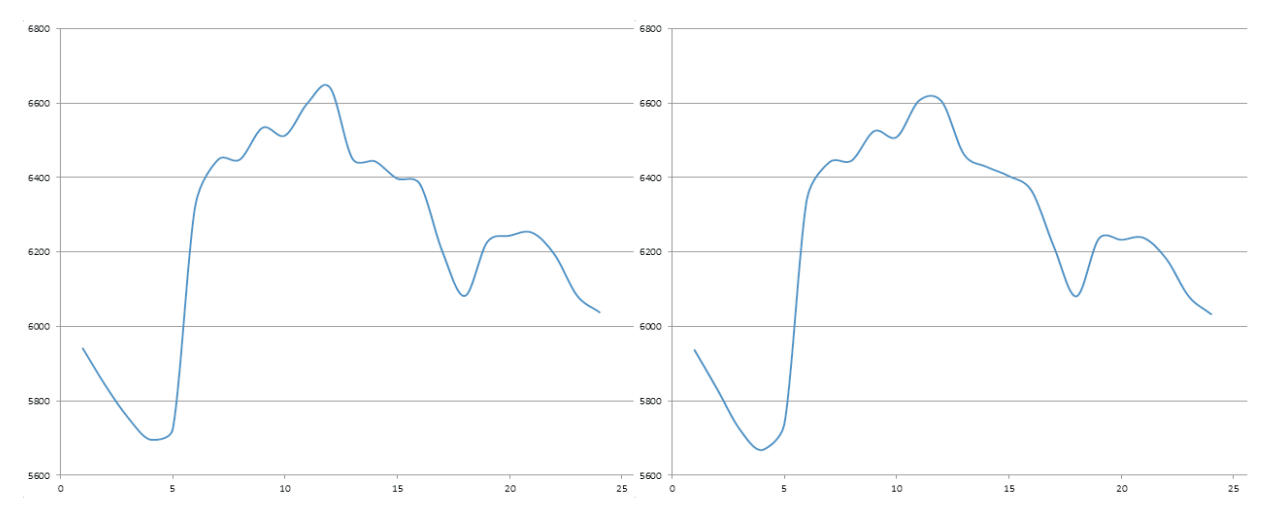

Fig. 8 Type daily diagram and standard of Wednesday. 
Garlík B., Křivan M.: Identification of type daily diagrams...

\begin{tabular}{|c|c|c|c|c|c|c|c|c|}
\hline \multicolumn{3}{|c|}{ Saturday } & \multicolumn{3}{|c|}{ Sunday } & \multicolumn{3}{|c|}{ Wednesday } \\
\hline TDD & standard & difference & TDD & standard & difference & TDD & standard & difference \\
\hline$[\mathrm{kW}]$ & [kW] & [\%] & {$[\mathrm{kW}]$} & {$[\mathrm{kW}]$} & {$[\%]$} & {$[\mathrm{kW}]$} & {$[\mathrm{kW}]$} & {$[\%]$} \\
\hline 5650 & 5648 & 0,04 & 5473 & 5475 & 0,04 & 5941 & 5937 & 0,07 \\
\hline 5600 & 5602 & 0,04 & 5424 & 5439 & 0,28 & 5842 & 5833 & 0,15 \\
\hline 5507 & 5523 & 0,29 & 5340 & 5346 & 0,11 & 5756 & 5725 & 0,54 \\
\hline 5453 & 5441 & 0,22 & 5314 & 5302 & 0,23 & 5696 & 5668 & 0,49 \\
\hline 5341 & 5362 & 0,39 & 5235 & 5240 & 0,10 & 5724 & 5738 & 0,24 \\
\hline 5411 & 5438 & 0,50 & 5259 & 5275 & 0,30 & 6323 & 6341 & 0,28 \\
\hline 5599 & 5586 & 0,23 & 5361 & 5360 & 0,02 & 6447 & 6441 & 0,09 \\
\hline 5749 & 5746 & 0,05 & 5518 & 5534 & 0,29 & 6449 & 6446 & 0,05 \\
\hline 5815 & 5813 & 0,03 & 5652 & 5648 & 0,07 & 6534 & 6525 & 0,14 \\
\hline 5894 & 5873 & 0,36 & 5754 & 5776 & 0,38 & 6513 & 6509 & 0,06 \\
\hline 5903 & 5885 & 0,31 & 5824 & 5800 & 0,41 & 6600 & 6607 & 0,11 \\
\hline 5822 & 5833 & 0,19 & 5703 & 5706 & 0,05 & 6642 & 6605 & 0,56 \\
\hline 5764 & 5744 & 0,35 & 5664 & 5649 & 0,27 & 6451 & 6462 & 0,17 \\
\hline 5759 & 5742 & 0,30 & 5661 & 5659 & 0,04 & 6444 & 6429 & 0,23 \\
\hline 5735 & 5740 & 0,09 & 5669 & 5665 & 0,07 & 6397 & 6404 & 0,11 \\
\hline 5744 & 5735 & 0,16 & 5644 & 5659 & 0,27 & 6383 & 6365 & 0,28 \\
\hline 5612 & 5613 & 0,02 & 5564 & 5555 & 0,16 & 6202 & 6214 & 0,19 \\
\hline 5578 & 5570 & 0,14 & 5545 & 5546 & 0,02 & 6082 & 6081 & 0,02 \\
\hline 5596 & 5591 & 0,09 & 5591 & 5583 & 0,14 & 6227 & 6236 & 0,14 \\
\hline 5611 & 5581 & 0,54 & 5586 & 5588 & 0,04 & 6244 & 6233 & 0,18 \\
\hline 5637 & 5631 & 0,11 & 5627 & 5624 & 0,05 & 6252 & 6238 & 0,22 \\
\hline 5606 & 5610 & 0,07 & 5640 & 5659 & 0,34 & 6193 & 6182 & 0,18 \\
\hline 5565 & 5566 & 0,02 & 5572 & 5579 & 0,13 & 6083 & 6081 & 0,03 \\
\hline 5528 & 5538 & 0,18 & 5735 & 5751 & 0,28 & 6038 & 6033 & 0,08 \\
\hline & & & & MFAN- & 0.17 & & MFAN- & 0.19 \\
\hline & $\mathrm{MAX}=$ & 0,54 & & MAX $=$ & 0,41 & & MAX $=$ & 0,56 \\
\hline
\end{tabular}

Tab. I Type daily diagram and standard.

of an ideal method, and is only suitable for the analysis of sufficiently distant homogenous clusters of approximately spherical shape, which reduces its utility value.

The cluster analysis of neural networks is thus, in comparison e.g. with the $k$ means method, completely universal in accordance with the nature of the location of objects in the examined file (it may also identify a completely uniform distribution of objects in an $n$-dimensional space), however the interpretation of its results partially depends on a subjective reading of the Kohonen map.

\section{References}

[1] Grossberg S.: The Adaptive Brain, North-Holland, 1987.

[2] Kohonen T.: The Self-Organizing Map, IEEE, 78, 9, 1990, pp. 1464-1480.

[3] R. Hecht-Nielsen R.: Counter-propagation networks, Appl. Opt. 26 (23), 1987, pp. 4979-4984.

[4] Hopfield J. J.: Artificial Neural Networks, IEEE Circuits \& Devices, 4, 3-10, 1988. 